\title{
A New Registration/Visualization Paradigm for CT-Fluoroscopy Guided RF Liver Ablation
}

\author{
Ruxandra Micu ${ }^{1,2}$, Tobias F. Jakobs ${ }^{3}$, Martin Urschler ${ }^{4}$, and Nassir Navab ${ }^{1}$ \\ ${ }^{1}$ Chair for Computer Aided Medical Procedures (CAMP), TU Munich, Germany \\ micu, navab@cs.tum. edu \\ ${ }^{2}$ Siemens Medical Solutions, Computed Tomography, Forchheim \\ ruxandra.micu.ext@siemens.com \\ ${ }^{3}$ Institute for Clinical Radiology, University of Munich, Grosshadern Hospital \\ tobias.jakobs@med.uni-muenchen.de \\ ${ }^{4}$ Institute for Computer Graphics and Vision, Graz University of Technology \\ urschler@icg.tu-graz.ac.at
}

\begin{abstract}
D-3D slice-to-volume registration for abdominal organs like liver is difficult due to the breathing motion and tissue deformation. The purpose of our approach is to ease CT-fluoroscopy (CT-fluoro) based needle insertion for the Radiofrequency Liver Ablation procedure using high resolution contrasted preoperative data. In this case, low signalto-noise ratio, absence of contrast and additional presence of needle in CT-fluoro makes it difficult to guarantee the solution of any deformable slice-to-volume registration algorithm. In this paper, we first propose a method for creating a set of ground truth (GT) simulation data based on a non-linear deformation of the CT-fluoro volume obtained from real patients. Second, we split the CT-fluoro image and apply intensity based rigid and affine registration to each section. We then propose a novel solution, which consists of intuitive visualization sequences of optimal sub-volumes of preinterventional data based on the registration results. Experiments on synthetic and real patient data and direct feedback of two interventionalists validate our alternative approach.
\end{abstract}

\section{Introduction}

Radiofrequency Ablation (RFA) of the liver is an interventional procedure applied to patients with unresectable primary liver tumors (HCC - hepatocellular carcinoma) and metastases. The percutaneous needle insertion is performed using different imaging guidance systems like CT, CT-fluoro, MRI or Ultrasound [1]. We are investigating the registration of the CT-fluoro slices with the preinterventional CT data-set (volume).

Related Work. Xu et al. 2] use 2D CT-fluoro to 3D CT volume intensity based registration for tracking pulmonary lesions. However, the tracking is based on a region rich in texture which cannot be applied in case of the liver. $\mathrm{Xu}$ et al. [3] estimate the deformation of CT slices of lung to CT volume by dividing the image into overlapping small disks. Note, that these lung applications did not use contrasted preinterventional data and have richer texture than liver. 
Combinations of features like vessels with intensity based methods for registering US slices to preoperative MR volumes also for RF liver ablation is reported in Penney et al. 4]. Unfortunately vessels are not visible in CT-fluoro slices in order to be used.

Major issues and main contribution. The complexity of this registration task is described below:

1. RFA is performed while the patient is breathing continuously, whereas the preinterventional CT volume is usually acquired during deep inspiration. One reason is because only in this breathing stage the pleura cavity can be appreciated to its full extension, and the other reason is that the patient can hold his breath for the imaging procedure more easily. Breathing instructions during the intervention are common. Once the needle is inserted into the target lesion breathing is usually impaired due to moderate pain and/or psychological reasons. A review regarding liver motion and deformation due to respiration was published in [5].

2. CT-fluoro slices have a lower signal-to-noise ratio compared to preinterventional CT data due to the lower radiation dose applied.

3. A single fluoro slice provides much less information than an entire CT volume. Futhermore it is thicker than preinterventional CT data.

4. The only anatomical features that could be used for liver registration are the vessels which are only seen in the contrast-enhanced preinterventional CT scan. Neither intrahepatic vessels nor metastases can be appreciated using CT-fluoro slices. Since primary liver cancer (HCC) is usually pretreated using transarterial chemoembolization (TACE), the used radiopaque embolic agent can be visualized in the non-enhanced CT-fluoro scan.

5. Registration needs to be done in real time.

In addition, no ground truth (GT) data are available. The generation of goldstandard data by incorporating fiducial markers [6] in the liver is, due to the minimal-invasive nature of this procedure, not feasible. This paper includes two main contributions. The first is the elaborate generation of a synthetic data set allowing precise analysis and testing of the registration solutions. For this purpose, approximate fluoro slice simulations were derived from preinterventional CT volume to evaluate registration algorithms. The creation of this synthetic data is described in section 3.1 .

Two experienced interventional radiologists independently defined planes in the preinterventional CT volume that they considered to be the best approximation to the corresponding fluoro slices. A variation considering the inter-observer agreement in defining corresponding slices was clearly observed. Partner physicians believe that there is also high chance for intra-observer disagreement, in case one interventionalist is asked to redefine the slices at a later time. Based on this finding and the major issues described above, we believe that a 2D-3D non-rigid registration solution precisely deforming the high quality contrasted 3D volume based on the single low quality not contrasted CT-fluoro cannot be guaranteed at the level required for medical procedures. 
The second contribution of this paper is therefore to introduce an alternative solution. Rigid and affine models do not capture the deformation [5]. However, we propose a novel solution, which consists of intuitive visualization sequences of optimal sub-volumes of preinterventional data based on rigid and affine registration results of splitted CT-fluoro slices. This sub-volume is automatically visualized next to the CT-fluoro and contains all the preinterventional information related to the fluoro slice. Details are presented in 2.2

\section{Methods}

\subsection{Registration}

Similarity measure. Due to the noise, the presence of the needle in the fluoro slices and the presence of contrast in the $3 \mathrm{D}$ volume a robust intensity based similarity measure, $f$ is chosen [7, where high differences in intensity are decreased. $\lambda$ controls the sensitivity of this bell shaped function.

$$
f=\sum_{i, j} \frac{\lambda^{2}}{\lambda^{2}+\left(I_{1}(i, j)-I_{2}(i, j)\right)^{2}}
$$

Registration details. The initial pose in the volume is set at the axial position where the fluoro slices are extracted in the deformed and noisy volume. This is analogous to initialization of real fluoroscopic slices using the DICOM header 'Image Position Patient' since the patient remains on the same table for the preinterventional CT volume scan and the intervention. RMS measurements between initial pose in the volume and defined pose by physicians vary between $26 \mathrm{~mm}-38 \mathrm{~mm}$, but this initial position proved to be best for convergence towards the target. A multiresolution approach is used to avoid local minima. The parameters for the rigid (6 DOF: 3 translations, 3 rotations) and affine transformation (12 DOF: rigid, 3 scalings, 3 shearings) of the CT volume, estimated at each resolution are used as initial values for the higher resolution. As optimization schemes we used the Best Neighbor and Powell-Brent. Real fluoro slices are additionally filtered with a Gaussian filter to reduce the noise and the artefacts of the needle.

\subsection{Volume Visualization Based on Piecewise Rigid and Affine Registration}

Dividing the liver into two parts, the upper part containing the ventral part of the liver and lower containing its dorsal part, enabled better results of the rigid/affine approximation. This is expected since the two parts undergo different level of movement and deformation. During expiration the rib cage volume decreases, thus increasing the pressure on the ventral aspect of the liver. The cut dividing the liver into upper and lower part is still manually positioned in the fluoro slice where the ligamentum falciforme is seen. The position of the cut is kept constant for all slices of one dataset. Sometimes during the intervention, the 
table is moved down allowing for more space for needle insertion. In this case it is needed to redefine the cut. Thus we are dividing the liver by a horizontal line into upper and lower part. These two parts are rigidly and affinely piecewise registered. In both models, rigid and affine, the estimated parameters by this piecewise registration define now the upper and lower planes. These resulting two planes should include a minimum volume with maximum information to be displayed in the operating room. The intersection line and angle between these two planes are calculated and then the target volume is shown as an animation by rotating $0.5^{\circ}$ at each visualization step around the intersection line, starting from the upper plane to the lower and back. Up to now the interventionalists move out of the operating room to the workstation outside to scroll through the high resolution volume in order to orientate better. Providing an additional display in the operating room with this small target volume visualization would be of great value.

\section{Results}

\subsection{Experiments with Simulated Fluoro Slices}

Simulation strategy The simulation strategy consists of adding realistic noise as if scanning with low dose [8] and applying a simulated breathing deformation as proposed in [9] to the pre-interventional datasets. We are using two routinely contrast-enhanced datasets from a 4 detector-row CT, Siemens Somatom Sensation4. Since they were acquired in deep inspiration breathhold, we apply the forces of deformation model such way that we simulate another volume in expiration stage, the rib cage contracting, while the liver is moving towards the heart. The two datasets were deformed with a translational force, representing the diaphragm movement, of $30 \mathrm{~mm}$ and with a radial force, representing the rib cage movement, of $5 \mathrm{~mm}$. Six axial slices are extracted from each noisy deformed volume, from all parts of the liver with $1 \mathrm{~cm}$ spacing in between. Fig. 11 shows one example from each dataset of real and simulated fluoro slices.

Noise addition For one dataset(dataset1) raw data are provided. This raw data is reconstructed again with Siemens Syngo IR Taskcard [8] twice. First time with parameters read from the DICOM header from the images reconstructed on the CT workstation in the hospital. Second time as if applying low dose to generate a noisy volume. Thus both volumes have the same field of view (FOV). For the second dataset (dataset2) raw data are not available. Therefore a software [10] is used to re-calculate the projections (raw data) of CT data. Noise is added to this raw data-set which is again reconstructed as noisy volume. Both datasets were downsampled in $\mathrm{x}$, y from $512 \times 512$ to $256 \times 256$ to fit fluoro slices, which have an image size of $256 \times 256$. The resulting volume sizes are for dataset1: $256 \times 256 \times 208$ voxels with a voxel size of $1.32 \times 1.32 \times 1.00 \mathrm{~mm}$, and for dataset 2 : $256 \times 256 \times 69$ with voxel size of $1.45 \times 1.45 \times 3.00 \mathrm{~mm}$. Both volumes have a CT slice thickness of $3 \mathrm{~mm}$. 


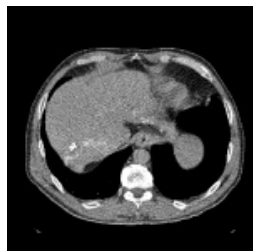

(a) real

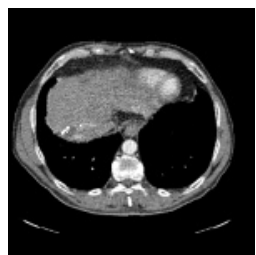

(b) $\operatorname{sim}$

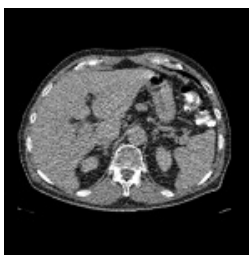

(c) real

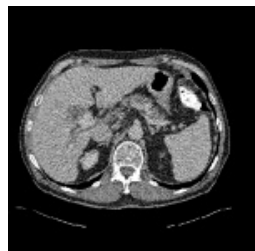

(d) $\operatorname{sim}$.

Fig. 1. 2D fluoro slices and simulations. (a) (b) - dataset1, (c), (d) - dataset2

Table 1. RMS[mm] Dataset1 - $256 \times 256 \times 208$

\begin{tabular}{|c||c|c|c|c|c|c||c|c|c|c|c|c|}
\hline \multicolumn{1}{|c||}{} & \multicolumn{9}{c||}{ Best Neighbor } & \multicolumn{5}{c|}{ Powell-Brent } \\
\hline \multicolumn{1}{|c||}{} & \multicolumn{3}{|c|}{ Rigid } & \multicolumn{3}{c||}{ Affine } & \multicolumn{3}{c|}{ Rigid } & \multicolumn{3}{c|}{ Affine } \\
\hline Slice & one & lower & upper & one & lower & upper & one & lower & upper & one & lower & upper \\
\hline 1 & 12.98 & 5.90 & 4.35 & 8.27 & 5.59 & 4.95 & 10.51 & 7.68 & 5.90 & 6.75 & 9.17 & 5.42 \\
\hline 2 & 11.08 & 5.77 & 4.61 & 9.86 & 5.83 & 4.95 & 10.36 & 6.96 & 6.43 & 6.95 & 6.24 & 7.39 \\
\hline 3 & 9.61 & 5.84 & 4.76 & 10.52 & 5.84 & 5.02 & 12.56 & 6.72 & 4.46 & 6.86 & 5.81 & 5.09 \\
\hline 4 & 9.69 & 5.95 & 4.90 & 9.72 & 6.17 & 4.89 & 9.19 & 7.21 & 8.17 & 7.57 & 6.25 & 8.36 \\
\hline 5 & 9.59 & 6.19 & 10.59 & 9.60 & 6.22 & 5.10 & 10.58 & 5.80 & 4.30 & 6.90 & 7.51 & 6.19 \\
\hline 6 & 8.20 & 6.53 & 4.73 & 9.51 & 6.43 & 4.85 & 8.53 & 6.37 & 7.75 & 8.07 & 5.88 & 5.36 \\
\hline \hline Mean & 10.19 & 5.84 & 9.58 & 5.49 & 10.29 & 6.48 & 7.18 & 6.56 \\
\hline
\end{tabular}

Registration details The registration starts at an initial resolution of $64 \times 64 \times$ 52 , increases to $128 \times 128 \times 104$ and finishes at $256 \times 256 \times 104$. Dataset2 is downsampled only in $\mathrm{x}$ and $\mathrm{y}$ direction due to the lower resolution along $\mathrm{z}$. We are first registering the entire fluoro slice to the volume. The fluoro slice is mapped back with the known displacement field in the original volume. This curved, ground truth, surface is used to calculate the RMS error to the corresponding voxels estimated by the rigid and affine registration. Then each slice is divided into lower and upper part. Tables 1 and 2 show the results of one plane versus the results of upper and lower plane. The RMS error between the fluoro surface and the registered planes decreases by almost half except for Powell-Brent affine registration. Fig. 2 shows the rigid registration result planes of slice $2 /$ dataset1 and 5/dataset1 with Best Neighbor optimization. The resulting planes of the registration are intersecting the ground truth fluoro surface. The upper and lower surface is better approximated by the 2 planes rather than by the one plane.

\subsection{Experiments with Real Fluoro Data}

Dataset2 is used here again. We selected 5 fluoro slices which were acquired during the intervention that contain needle, the tumor and different parts of the liver beeing imaged often during the intervention. For the 5 fluoro slices $(256 \times 256,1.48 \times 1.48 \mathrm{~mm}$, slice thickness $4 \mathrm{~mm})$ we have independently defined 


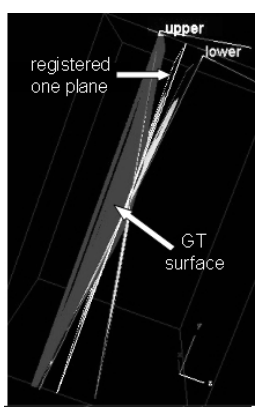

(a) $3 \mathrm{D}$ View

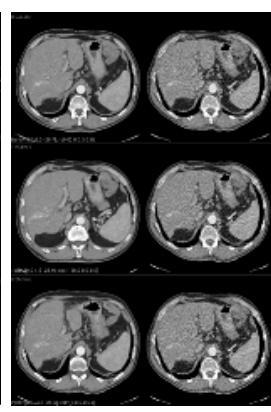

(b) 2D Views

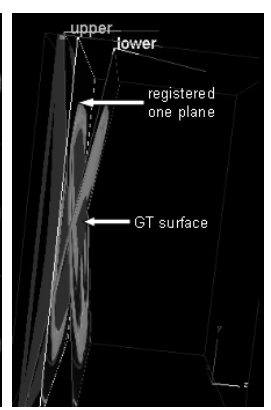

(c) 3D View

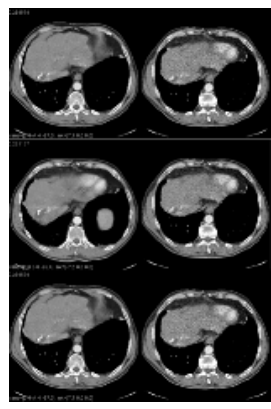

(d) $2 \mathrm{D}$ Views

Fig. 2. 3D view showing GT surface and registered upper, lower and one plane. 2D views showing from top to bottom the registered one plane, upper and lower.

Table 2. RMS[mm] Dataset2 - $256 \times 256 \times 69$

\begin{tabular}{|c|c|c|c|c|c|c|c|c|c|c|c|c|}
\hline & \multicolumn{6}{|c|}{ Best Neighbor } & \multicolumn{6}{|c|}{ Powell-Brent } \\
\hline & \multicolumn{3}{|c|}{ Rigid } & \multicolumn{3}{|c|}{ Affine } & \multicolumn{3}{|c|}{ Rigid } & \multicolumn{3}{|c|}{ Affine } \\
\hline Slice & one & lower & upper & one & lower & upper & one & lower & upper & one & lower & upper \\
\hline 1 & 10.66 & 6.78 & 8.69 & 11.19 & 6.70 & 5.46 & 10.28 & 8.85 & 10.27 & 10.02 & 8.45 & 9.19 \\
\hline 2 & 15.97 & 12.89 & 12.23 & 16.64 & 7.46 & 7.52 & 16.34 & 8.06 & 7.49 & 12.02 & 11.67 & 9.86 \\
\hline 3 & 15.04 & 7.90 & 9.32 & 15.68 & 6.94 & 9.04 & 16.44 & 8.50 & 8.23 & 7.45 & 9.13 & 15.01 \\
\hline 4 & 10.25 & 6.594 & 4.87 & 13.51 & 6.57 & 5.66 & 11.37 & 7.07 & 6.14 & 9.87 & 6.45 & 8.46 \\
\hline 5 & 11.55 & 8.50 & 6.86 & 18.48 & 8.35 & 5.84 & 11.73 & 8.52 & 9.77 & 10.66 & 9.39 & 10.93 \\
\hline 6 & 10.20 & 7.72 & 5.59 & 10.08 & 7.59 & 6.53 & 10.26 & 7.86 & 8.86 & 7.87 & 9.53 & 5.85 \\
\hline Mean & 12.28 & 8 & .15 & 14.26 & & & 12.74 & & 30 & 9.65 & & 49 \\
\hline
\end{tabular}

with two physicians the planes that they consider the closest to the respective fluoro slices. The manual navigation included 6 DOF, 3 for translation and 3 for rotation. This dataset has a significant liver motion and deformation so it was hard to define the planes even by experts with manual registration. The other dataset which was used here, dataset3 $(256 \times 256 \times 158,1.29 \times 1.29 \times 1.5 \mathrm{~mm})$ was acquired in shallow breathing. The liver motion is not so significant as in dataset2. We selected 3 fluoro slices $(256 \times 256,1.29 \times 1.29 \mathrm{~mm}$, slice thickness $4 \mathrm{~mm}$ ): one with needle and tumor, one with tumor, one with the liver next to the heart. The variation between the defined slices by the physicians is shown below. Only liver voxels contribute to the RMS calculation.

\begin{tabular}{|c||c|c|c|c|c||c|c|c|}
\hline \multicolumn{1}{|c||}{} & \multicolumn{5}{c||}{ Dataset2 } & \multicolumn{3}{c|}{ Dataset3 } \\
\hline Slice & 1 & 2 & 3 & 4 & 5 & 1 & 2 & 3 \\
\hline RMS[mm] & 17.16 & 10.25 & 5.79 & 16.50 & 19.68 & 4.12 & 7.05 & 9.08 \\
\hline
\end{tabular}

The amount of voxels at each position $\mathbf{p}$ included in the volume of interest $V$ defined by the upper plane: $\mathbf{n}_{\mathbf{u}}, d_{u}$ and lower plane: $\mathbf{n}_{\mathbf{l}}, d_{l}$, satisfy following inequality:

$$
\left(\mathbf{p n}_{\mathbf{l}}^{T}-d_{l}\right)\left(\mathbf{p n}_{\mathbf{u}}^{T}-d_{u}\right)<0
$$




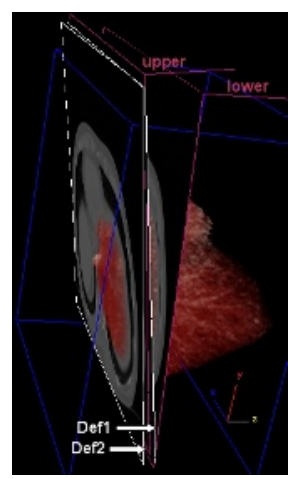

(a) ds2-slice3

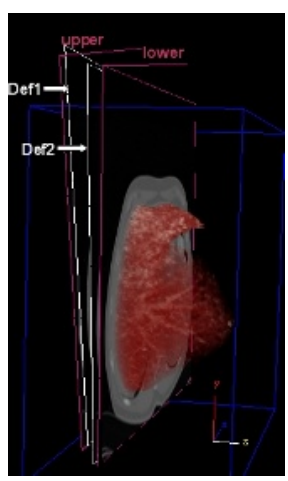

(b) ds2-slice1

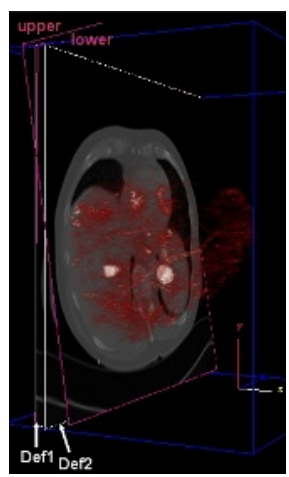

(c) ds3-slice1

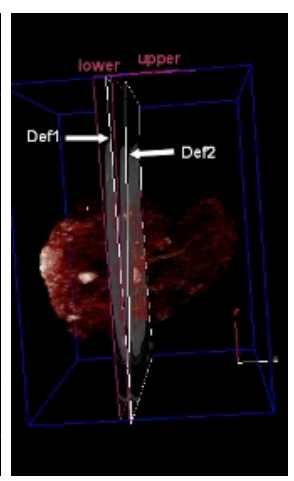

(d) ds3-slice2

Fig. 3. Configurations of registered upper and lower plane, defined slices Def1, Def2 by the physicians and rendered liver surface for rigid registration with Powell-Brent optimization scheme. Percentage of intersection between liver voxels and the volume defined by the two physicians: (a) Def1 30\%, Def2 0\%, (b) Def1 100\%, Def2 100\%, (c) Def1 100\%, Def2 79\%, (d) Def1 79\%, Def2 0\%

Table 3. Dataset2 - \% of included liver voxels for each defined slice in the sub-volume

\begin{tabular}{|c||c|c|c|c|c|c||c|c|c|c|c|c|}
\hline \multicolumn{1}{|c||}{} & \multicolumn{8}{c|}{ Percentage of Voxels included in VOI } \\
\hline \multicolumn{1}{|c||}{} & \multicolumn{8}{c||}{ Rigid Powell } & \multicolumn{5}{c|}{ Affine Powell } \\
\hline Slice & 1 & 2 & 3 & 4 & 5 & Mean & 1 & 2 & 3 & 4 & 5 & Mean \\
\hline \%Def1 & 100 & 94.45 & 30.20 & 79.11 & 65.5 & 75.26 & 100 & 81.07 & 65.32 & 59.19 & 67.91 & 67.91 \\
\hline \%Def2 & 100 & 96.09 & 0 & 74.52 & 11.81 & 60.01 & 96.12 & 87.87 & 2.82 & 61.17 & 14.13 & 55.73 \\
\hline & \multicolumn{9}{|c||c||c|c|c|c|c|c|c|c|c|c|c|}{} \\
\hline \%Def1 & 72.55 & 73.57 & 44.00 & 71.00 & 78.81 & 68.95 & 88.14 & 24.44 & 5.58 & 68.89 & 65.50 & 54.00 \\
\hline \%Def2 & 49.58 & 76.62 & 0 & 70.00 & 100 & 62.15 & 72.69 & 27.75 & 7.77 & 70.14 & 19.91 & 41.21 \\
\hline
\end{tabular}

Table 4. Dataset3 - \% of included liver voxels for each defined slice in the sub-volume

\begin{tabular}{|c||c|c|c|c||c|c|c|c|}
\hline \multicolumn{1}{|c||}{} & \multicolumn{5}{c|}{ Percentage of Voxels included in VOI } \\
\hline \multicolumn{1}{|c||}{} & \multicolumn{4}{c||}{ Rigid Powell } & \multicolumn{4}{c|}{ Affine Powell } \\
\hline Slice & 1 & 2 & 3 & Mean & 1 & 2 & 3 & Mean \\
\hline \% Def1 & 99.60 & 78.93 & 100 & 91.37 & 98.88 & 100 & 45.63 & 79.96 \\
\hline \%Def2 & 72.51 & 0 & 100 & 55.70 & 63.79 & 64.58 & 73.55 & 67.71 \\
\hline & \multicolumn{3}{|c||}{ Rigid BestNeighbor } & Affine BestNeighbor \\
\hline \%Def1 & 5.59 & 52.82 & 77.90 & 51.03 & 44.64 & 97.49 & 37.99 & 63.63 \\
\hline \%Def2 & 69.45 & 0 & 83.16 & 48.66 & 69.3 & 6.22 & 53.09 & 39.77 \\
\hline
\end{tabular}

Tables 3 and 4 show the percentage of liver voxels of the defined slices Def1 and Def2 by the two phyisicians, intersecting the visualized minimum volume. For real data the rigid model with Powell-Brent optimization works best. Particular slice 3 of dataset2 (ds2) poses difficulties for automatic registration due to a big $\operatorname{rotation}\left(\approx 12^{\circ}\right)$ about the $\mathrm{x}$ axis compared to the initial pose. But visual 
inspection of the resulting registration and angle calculations of both defined slices to the closest registered plane of the minimum volume for this case (Def2: $0.77^{\circ}$ and Def1: $1.96^{\circ}$ ), show that they lie very close to the volume. Fig. 3 (a) shows this configuration, (b) shows an ideal configuration as for slice 1. For slice 2 of dataset3 (ds3) we also calculated the angles of the defined slices to the registered closest plane. Additionally, the RMS error between liver voxels that are not included in the volume and the closest registered plane was calculated (Def1: $1.38^{\circ}$, RMS 1.92mm, Def2: $2.52^{\circ}$, RMS 5.65mm). Both RMS errors lie under RMS variation of Def1 and Def2 for this slice. This configuration is shown in fig. 3 (d).

\section{Conclusion}

In this paper we first presented major issues in providing a reliable 2D-3D registration between preinterventional contrasted CT and interventional noncontrasted CT-fluoro. We then presented a method for creation of GT data based on approximation of breathing and realistic noise models where we could evaluate our two part visualization-registration algorithm. This two part visualizationregistration was then applied to registration with real CT-fluoro slices. In this way, we estimated the sub-volume of the preoperative CT data where pixels of the CT-fluoro image have originated from. This small size sub-volume of the high resolution preinterventional contrasted $\mathrm{CT}$ data is thus presented in the operating room as a short animation sequence next to the acquired fluoro slice. Results of the synthetic and real experiments and the feedback from two interventional radiologists confirmed the value of this alternative approach.

Acknowledgements. This research was funded by Siemens Medical Solutions, Germany within the VICORA project. The authors would like to thank in particular to Rainer Graumann, Michael Sühling (Siemens Medical Solutions) and Prof. Reiser (Institute for Clinical Radiology, Grosshadern) for their continuous support.

\section{References}

1. Friedman, M., Mikityansky, I., Kam, A., Libutti, S., Walther, M.M., Neeman, Z., Locklin, J.K., Wood, B.J.: Radiofrequency ablation of cancer. CardioVascular and Interventional Radiology (2004)

2. Xu, S., Fichtinger, G., Taylor, R.H., Cleary, K.R.: 3d motion tracking of pulmonary lesions using ct fluoroscopy images for robotically assisted lung biopsy. In: Medical Imaging 2004: Visualization, Image-Guided Procedures, and Display. Volume 5367., Galloway, R.L., Jr. (ed.) (2004) 394-402

3. Xu, S., Taylor, R., Fichtinger, G., Cleary, K.: Lung deformation estimation and four-dimensional ct lung reconstruction. In: Proc. Int'l Conf. Medical Image Computing and Computer Assisted Intervention (MICCAI). Volume 3750 of Lecture Notes in Computer Science., Springer (2005) 312-319 
4. Penney, G., Blackall, J., Hamady, M., Sabharwal, T., Adam, A., Hawkes, D.: Registration of freehand $3 \mathrm{~d}$ ultrasound and magnetic resonance liver images. Medical Image Analysis 8 (2004) 81-91

5. Clifford, M., Banovac, F., Levy, E., Cleary, K.: Assessment of hepatic motion secondary to respiration for computer assisted interventions. Computer Aided Surgery 7 (2002) 291-299

6. Russakoff, D., Rohlfing, T., A. Ho, D.K., Shahidi, R., J.R. Adler, J., C. R. Maurer, J.: Evaluation of intensity-based $2 \mathrm{~d}-3 \mathrm{~d}$ spine image registration using clinical goldstandard data. In: Workshop on Biomedical Image Registration (WBIR). (2003)

7. Penney, G.P., Weese, J., Little, J.A., Desmedt, P., Hill, D.L.G., Hawkes, D.J.: A comparision of similarity measures for use in $2 \mathrm{~d}-3 \mathrm{~d}$ medical image registration. IEEE Transactions on Medical Imaging (TMI) 17 (1998) 586-595

8. Ulzheimer, S., Leidecker, C.: Syngo explorer image reconstruction(ir) taskcard, bericht zur validierung der option 'addition von rauschen'. VAMP Verfahren und Apparate der Medizinischen Physik 8 (2004)

9. Urschler, M., Bischof, H.: Assessing breathing motion by shape matching of lung and diaphragm surfaces. In: Medical Imaging 2005: Visualization, Image-Guided Procedures, and Display. SPIE, Galloway, R.L., Jr. (ed.) (2005) 440-452

10. Wessling, J., Fischbach, R., Esseling, R., Raupach, R., Heindel, W.: Effect of dose reduction and noise reduction filters on detection of liver lesions using multidetector row ct. In: RSNA. (2005) 